\title{
A narrative review of machine learning as promising revolution in clinical practice of scoliosis
}

\author{
Kai Chen ${ }^{1 \#}$, Xiao Zhai ${ }^{1 \#}$, Kaiqiang Sun ${ }^{2 \#}$, Haojue Wang ${ }^{3 \#}$, Changwei Yang ${ }^{1}$, Ming Li ${ }^{1}$ \\ ${ }^{1}$ Department of Orthopedics, Shanghai Changhai Hospital, Shanghai, China; ${ }^{2}$ Department of Orthopedics, Shanghai Changzheng Hospital, \\ Shanghai, China; ${ }^{3}$ Basic medicine college, Navy Medical University, Shanghai, China \\ Contributions: (I) Conception and design: C Yang; (II) Administrative support: M Li; (III) Provision of study materials or patients: K Chen; (IV) \\ Collection and assembly of data: X Zhai; (V) Data analysis and interpretation: K Sun, H Wang; (VI) Manuscript writing: All authors; (VII) Final \\ approval of manuscript: All authors. \\ "These authors contributed equally to this work. \\ Correspondence to: Changwei Yang, MD; Ming Li, MD. Department of Orthopedics, Shanghai Changhai Hospital, No. 168, Changhai Road, \\ Shanghai 200433, China. Email: changwei_y@qq.com; limingch0103@126.com.
}

\begin{abstract}
Machine learning (ML), as an advanced domain of artificial intelligence (AI), is progressively changing our view of the world. By implementing its algorithms, our ability to detect previously undiscoverable patterns in data has the potential to revolutionize predictive analytics. Scoliosis, as a relatively specialized branch in the spine field, mainly covers the pediatric, adult and the elderly populations, and its diagnosis and treatment remain difficult. With recent efforts and interdisciplinary cooperation, ML has been widely applied to investigate issues related to scoliosis, and surprisingly augment a surgeon's ability in clinical practice related to scoliosis. Meanwhile, ML models penetrate in every stage of the clinical practice procedure of scoliosis. In this review, we first present a brief description of the application of ML in the clinical practice procedures regarding scoliosis, including screening, diagnosis and classification, surgical decision making, intraoperative manipulation, complication prediction, prognosis prediction and rehabilitation. Meanwhile, the ML models and specific applications adopted are presented. Additionally, current limitations and future directions are briefly discussed regarding its use in the field of scoliosis. We believe that the implementation of ML is a promising revolution to assist surgeons in all aspects of clinical practice related to scoliosis in the near future.
\end{abstract}

Keywords: Scoliosis; machine learning (ML); revolution; clinical practice

Submitted Jul 24, 2020. Accepted for publication Nov 27, 2020.

doi: $10.21037 /$ atm-20-5495

View this article at: http://dx.doi.org/10.21037/atm-20-5495

\section{Introduction}

The past few decades have seen a massive increase in the use of artificial intelligence (AI). Machine learning (ML) is an advanced branch of AI that allows computer algorithms to learn patterns by studying data directly without being explicitly programmed. ML is similar to the human neural network that can learn, make decisions, communicate, and adapt to changing circumstances (1). Generally, ML is categorized into three primary methods: supervised, unsupervised, and reinforcement learning (2). Also, ML lends itself well to image processing due to its extremely high classification performance, and there have been a few studies regarding its applications to medical imaging (3). By implementing such methods, it is possible to revolutionize predictive analytics for previously undiscoverable patterns by leveraging existing big data, and it might have widespread implications for medical research.

Compared to other industries, healthcare is relatively slow in adopting AI (4). The incredible complexity of healthcare delivery is strangely what makes it a very fertile ground for the application of AI (5). However, technology is constantly changing throughout clinical practice, 
including how doctors interact with patients, diseases and their implements, the approach of information delivery, how the resultant interpretation is used to aid physicians, and postoperative evaluation and rehabilitation (6). The first attempts to introduce AI in spine surgery dated back to the advert of general-purpose computers during the Second World War and became available for nonmilitary use in the 1950s, which have been providing new insights into previous untapped and rapidly growing sources of data for reasoning and deciding. Nowadays, ML is entering the realm of medicine at an increasing pace and increasingly being used to investigate spine-related issues, especially in radiological imaging. For example, Jamaludin et al. $(7,8)$ ever proposed a ML based system (Oxford SpineNet software system) for automatically analyzing spinal T2 MRI scans acquired from a DICOM (Digital Imaging and Communications in Medicine) file to evaluate Pffirrmann grades, modic changes, and spinal stenosis, and found that the system can be beneficial in aiding clinical diagnoses in terms of objectivity of gradings and the speed of analysis. In addition, ML is also applied in the outcome prediction of treatments (9). Arvind et al. (10) included 20,879 patients following anterior cervical discectomy and fusion and found that ANN have the greatest sensitivity in predicting mortality and postoperative complications. Similarly, Kim et al. (11) also reported the superiority of ML in identifying risk factors of developing complications following posterior lumbar spine fusion. Other applications of ML in spine surgery included diagnosis and assessment of spinal disease progression, decision-making in the treatment of spine degenerative diseases, and preoperative planning and intraoperative assistance (12).

Scoliosis, as a major composition of complex threedimensional spine deformity, mainly covers the pediatric, adult and the elderly populations, and its diagnosis and treatment remain difficult. For decades, surgeons in this field relied on the established literature, extensive training, and clinical judgment to counsel patients regarding the risks and benefits of surgery; often, the most accurate information was based on their overall personal clinical experience and lacked patient-specific characteristics. With recent efforts and interdisciplinary cooperation, ML has been widely applied to investigate issues related to scoliosis $(2,13)$. In this realm of enhanced technology and digital innovation, we are witnesses to this revolution and transformation. Surgeons in this field have been quickly adapting and refining these new technologies and integrated them into their clinical practice. The current trends in scoliosis are about digitization, ML, and smart robotics (14). ML models can meaningfully augment a surgeon's ability in clinical practice related to scoliosis. As a result, there has been more considerable interest in the literature and academic forums about the utilization of ML in various branches in this field.

In this article, we did a wide search in the PubMed and Embase databases, and the searching strategy applied was as follows: Machine Learning [All Fields] AND ("Scoliosis" [MeSH Terms] OR "Spine Deformity" [All Fields]). Based on the cited references, we enlarged the search range and adopted the useful publications. By means of this narrative literature review, we aim to raise awareness of the current achievements and potential applications of ML in the field of scoliosis. It is vitally important to have the ability to establish an accurate clinical practice procedure, including screening, diagnosis and classification, surgical decision making, intraoperative manipulation, complication prediction, prognosis prediction and rehabilitation (Figure 1), in selecting the most appropriate management strategy for disorders in this field, as shown in Table 1. Therefore, this paper firstly presents ML and its recent advances, which might have a dramatical impact on clinical practice procedures in treating scoliosis. We present the following article in accordance with the Narrative Review reporting checklist (available at http://dx.doi.org/10.21037/atm-205495).

\section{Screening, diagnosis, and classification}

Currently, imaging identification has become a tremendous field of ML. Given the characteristics of a regular sequence of the normal spine and the irregularity of scoliosis, $\mathrm{ML}$ has superiority in identifying this disorder. To efficiently screen and diagnose a patient with scoliosis, ML has been transformative.

Screening may detect scoliosis earlier than it would be clinically detected. With early detection, most cases can be controlled with the lower costs and better efficacy. In addition, accurate diagnosis with ML can help surgeons avoid misjudgment. Early in 2000, Jaremko et al. (15) was the first to use neural networks to correlate spine and rib deformities in scoliosis. The investigators compared artificial neural networks (ANNs) and linear regression to predict rib rotation, with the results that ANNs averaged $60 \%$ correct predictions compared to $34 \%$ for linear regression analysis. These data lend credence to using ANNs in future work on the prediction of scoliotic spinal deformities from 


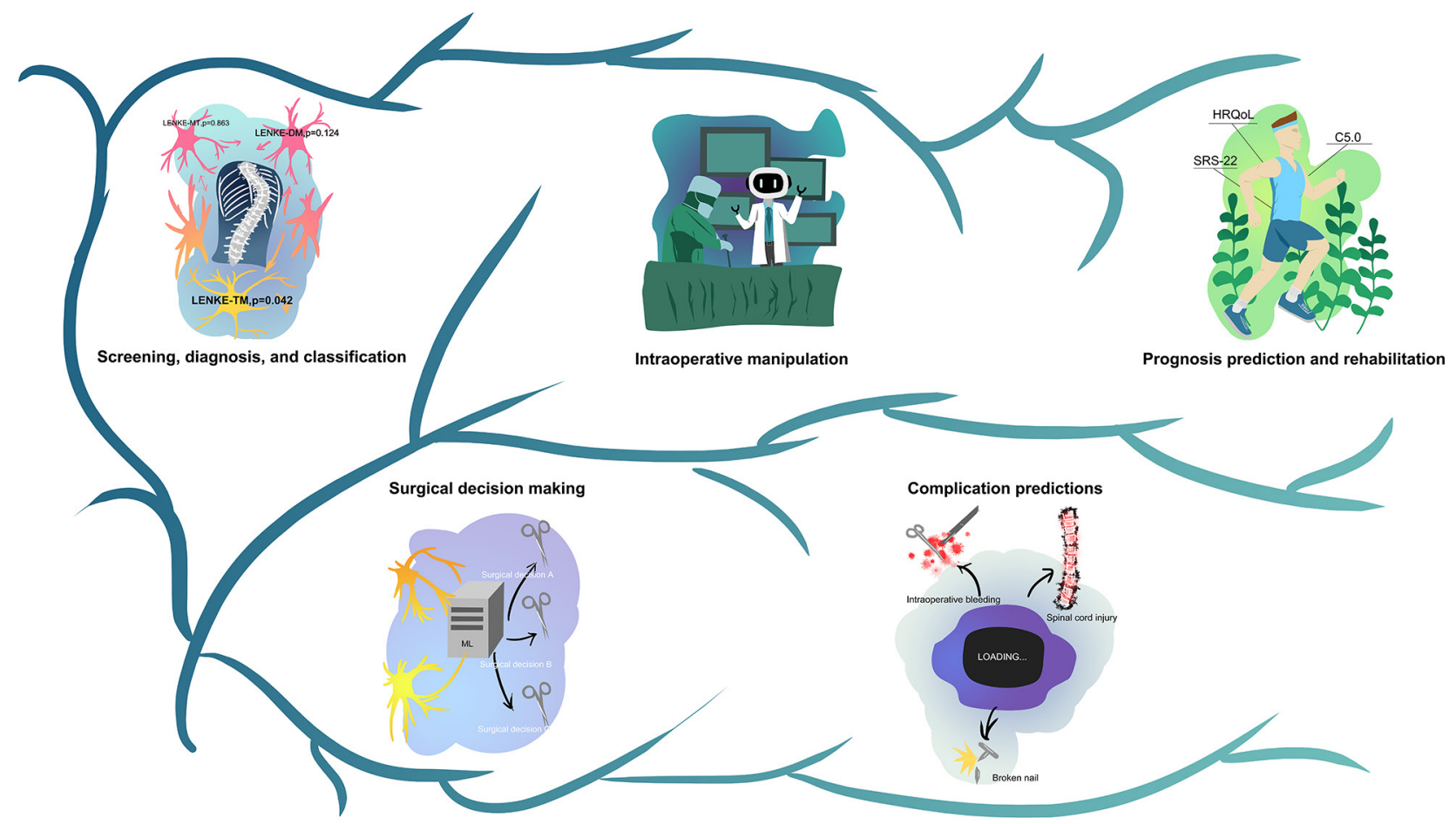

Figure 1 The demonstration of the application of ML in the clinical practice of scoliosis.

Table 1 The application of machine learning in the clinical practice of scoliosis

\begin{tabular}{|c|c|c|c|c|c|}
\hline Study & Year & Number of data & Algorithms applied & Objectives & Outcome presentation \\
\hline Jaremko (15) & 2000 & 57 curves & ANN, linear regression & Predicting rib deformity & $\begin{array}{l}\text { ANN showed good sensitivity and } \\
\text { PPV }\end{array}$ \\
\hline Jaremko (16) & 2001 & $\begin{array}{l}65 \text { radiograph- } \\
\text { pairs }\end{array}$ & ANN & $\begin{array}{l}\text { Estimating spinal deformity } \\
\text { from torso surface cross } \\
\text { sections }\end{array}$ & $\begin{array}{l}\text { Distinguished a Cobb angle greater } \\
\text { than } 30^{\circ} \text { with excellent sensitivity and } \\
\text { specificity }\end{array}$ \\
\hline Ramirez (17) & 2006 & 111 patients & SVM & $\begin{array}{l}\text { Assessing the severity of IS } \\
\text { from surface topography }\end{array}$ & Satisfactory accuracy in testing \\
\hline $\operatorname{Lin}(18)$ & 2008 & $\begin{array}{l}37 \text { spinal } \\
\text { deformity } \\
\text { patterns }\end{array}$ & $\begin{array}{l}\text { A multilayer feed-forward, } \\
\text { back-propagation ANN }\end{array}$ & $\begin{array}{l}\text { Identifying the King } \\
\text { classification patterns of the } \\
\text { scoliosis }\end{array}$ & $\begin{array}{l}\text { Excellent identification rate for one or } \\
\text { two hidden layers }\end{array}$ \\
\hline Menon (21) & 2014 & 62 cases of AIS & - & $\begin{array}{l}\text { Retrieving images of similar } \\
\text { cases of AIS }\end{array}$ & - \\
\hline
\end{tabular}

Table 1 (continued) 
Table 1 (continued)

\begin{tabular}{|c|c|c|c|c|c|}
\hline Study & Year & Number of data & Algorithms applied & Objectives & Outcome presentation \\
\hline Thong (23) & 2016 & 663 patients & $\begin{array}{l}\text { A stacked autoencoder } \\
\text { consisting of a specific } \\
\text { ANN architecture; } \\
\text { k-means++ clustering } \\
\text { algorithm }\end{array}$ & $\begin{array}{l}\text { Performing a 3D } \\
\text { morphological analysis of } \\
\text { spine }\end{array}$ & $\begin{array}{l}\text { The model can simplify the complex } \\
\text { nature of 3D spine models as well as } \\
\text { preserve the intrinsic properties }\end{array}$ \\
\hline $\begin{array}{l}\text { Bertoncelli } \\
\text { (24) }\end{array}$ & 2018 & 120 patients & $\begin{array}{l}\text { A predictive model based } \\
\text { on a logistic regression } \\
\text { algorithm }\end{array}$ & $\begin{array}{l}\text { Validating the performance of } \\
\text { a clinical prediction model }\end{array}$ & $\begin{array}{l}\text { Good average accuracy, sensitivity, } \\
\text { and specificity }\end{array}$ \\
\hline $\begin{array}{l}\text { García-Cano } \\
(26)\end{array}$ & 2018 & $\begin{array}{l}9623 D \text { spine } \\
\text { models }\end{array}$ & $\begin{array}{l}\text { Dynamic ensemble } \\
\text { selection }\end{array}$ & Assessing curve types & $\begin{array}{l}\text { A mean accuracy of } 0.7766 \text { and a } \\
\text { mean log loss of } 0.5623\end{array}$ \\
\hline Greer (27) & 2018 & 10,000 images & $\begin{array}{l}\text { A convolutional network } \\
\text { and a second fully } \\
\text { connected network }\end{array}$ & $\begin{array}{l}\text { Diagnosing scoliosis using } \\
\text { a self-contained ultrasound } \\
\text { device }\end{array}$ & $\begin{array}{l}\text { The mean error is } 2.0^{\circ} \text {, the standard } \\
\text { deviation is } 3.7^{\circ} \text {, and the } 95 \text { th } \\
\text { percentile error is } 5.8^{\circ}\end{array}$ \\
\hline Wu (28) & 2018 & $\begin{array}{l}526 \text { X-ray } \\
\text { images }\end{array}$ & $\begin{array}{l}\text { A novel multi-view } \\
\text { correlation network }\end{array}$ & $\begin{array}{l}\text { Automatically quantitative } \\
\text { estimating spinal curvature }\end{array}$ & $\begin{array}{l}4.04^{\circ} \mathrm{CMAE} \text { in anteroposterior (AP) } \\
\text { Cobb angle and } 4.07^{\circ} \mathrm{CMAE} \text { in lateral } \\
\text { (LAT) Cobb angle estimation }\end{array}$ \\
\hline $\begin{array}{l}\text { Watanabe } \\
\text { (31) }\end{array}$ & 2019 & $\begin{array}{l}1,996 \text { pairs of } \\
\text { moiré images } \\
\text { and standing } \\
\text { whole-spine } \\
\text { radiographs }\end{array}$ & CNN & $\begin{array}{l}\text { Estimating spinal alignment } \\
\text { from moiré images }\end{array}$ & $\begin{array}{l}\text { The MAE per person between the } \\
\text { Cobb angle measured by doctors and } \\
\text { the estimated Cobb angle was } 3.42^{\circ} \text {. } \\
\text { The MAE was } 4.38^{\circ} \text { in normal spines, } \\
3.13^{\circ} \text { in spines with a slight deformity, } \\
\text { and } 2.74^{\circ} \text { in spines with a mild to } \\
\text { severe deformity. The MAE of the } \\
\text { angle of vertebral rotation was } \\
2.9^{\circ} \pm 1.4^{\circ} \text { and was smaller when the } \\
\text { deformity was milder }\end{array}$ \\
\hline Wang (32) & 2019 & 526 X-rays & Multiview extrapolation net & $\begin{array}{l}\text { Accurately and automatically } \\
\text { estimating Cobb angle }\end{array}$ & $\begin{array}{l}7.81 \text { and } 6.26 \text { CMAE in AP and LAT } \\
\text { angle estimation }\end{array}$ \\
\hline Horng (33) & 2019 & 35 images & CNN & $\begin{array}{l}\text { Proposing an automatic } \\
\text { system to measure spine } \\
\text { curvature }\end{array}$ & $\begin{array}{l}\text { Excellent results of ICC and Pearson } \\
\text { correlation coefficients }\end{array}$ \\
\hline
\end{tabular}

Table 1 (continued) 
Table 1 (continued)

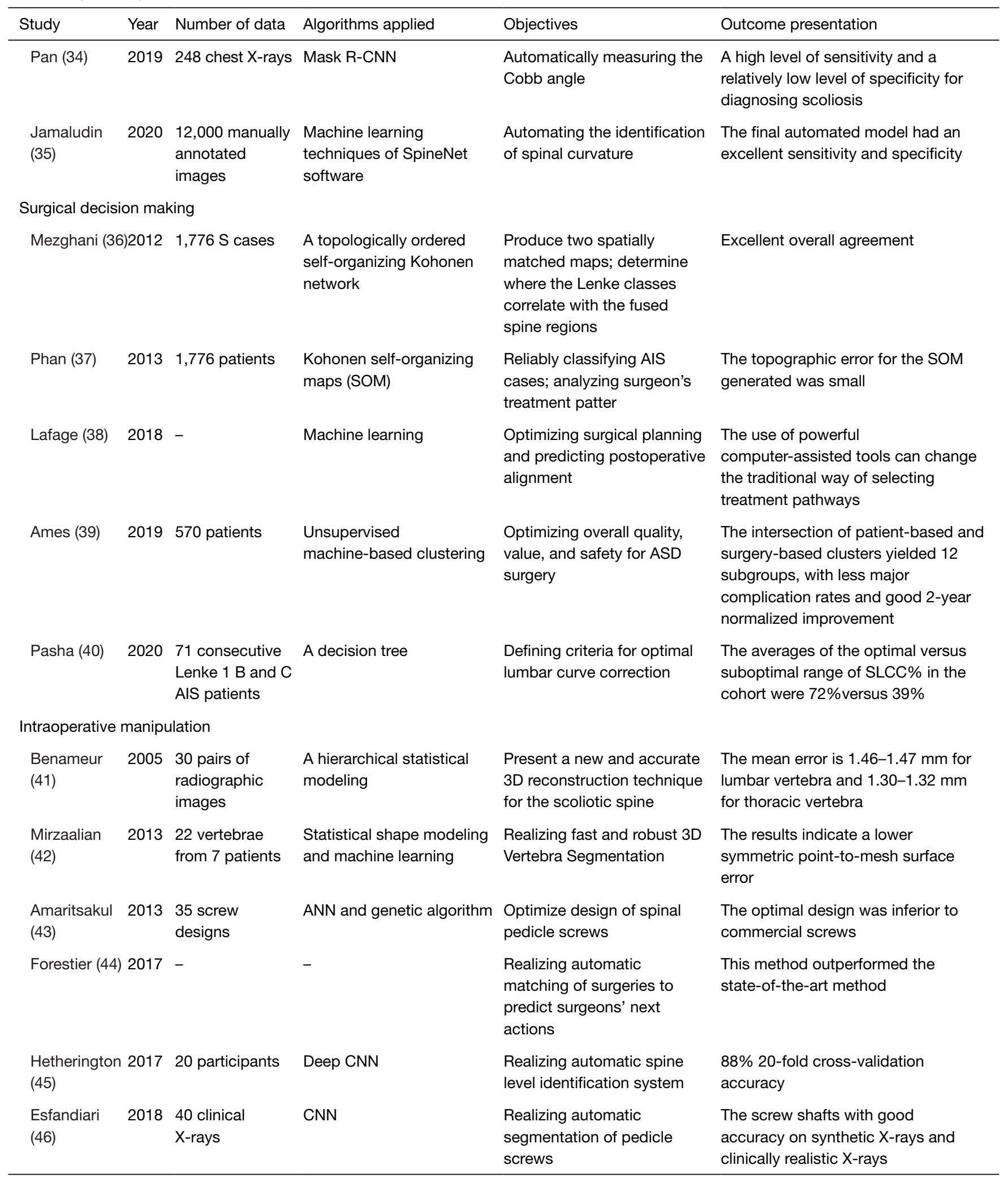

Table 1 (continued) 
Table 1 (continued)

\begin{tabular}{|c|c|c|c|c|c|}
\hline Study & Year & Number of data & Algorithms applied & Objectives & Outcome presentation \\
\hline Huo (49) & 2020 & $\begin{array}{l}400 \text { individual } \\
\text { vertebral models }\end{array}$ & $\begin{array}{l}\text { A modified PointNet model } \\
(\mathrm{CNN})\end{array}$ & $\begin{array}{l}\text { Automatically recognizing the } \\
\text { vertebral pedicle in individual } \\
\text { vertebral models and } \\
\text { drawing pedicle contours }\end{array}$ & $\begin{array}{l}\text { The final results can be used to } \\
\text { simulate the operation of pedicle } \\
\text { screw implantation and to provide a } \\
\text { reference }\end{array}$ \\
\hline \multicolumn{6}{|c|}{ Complication predictions } \\
\hline Scheer (50) & 2016 & 510 patients & $\begin{array}{l}\text { An ensemble of } \\
\text { decision trees using the } \\
\text { C5.0 algorithm with } 5 \\
\text { different bootstrapped } \\
\text { models }\end{array}$ & $\begin{array}{l}\text { To create a preoperative } \\
\text { predictive model for proximal } \\
\text { junction failure (PJF) }\end{array}$ & $\begin{array}{l}\text { The overall model accuracy indicated } \\
\text { a good model fit }\end{array}$ \\
\hline Scheer (51) & 2017 & $\begin{array}{l}557 \text { ADS } \\
\text { patients }\end{array}$ & $\begin{array}{l}\text { An ensemble of decision } \\
\text { trees utilizing the C5.0 } \\
\text { algorithm with } 5 \text { different } \\
\text { bootstrapped models }\end{array}$ & $\begin{array}{l}\text { To create a preoperative } \\
\text { predictive model for major } \\
\text { complications }\end{array}$ & $\begin{array}{l}\text { The overall model accuracy indicated } \\
\text { a very good model fit }\end{array}$ \\
\hline Pellisé (54) & 2019 & $\begin{array}{l}1,612 \text { ASD } \\
\text { patients }\end{array}$ & $\begin{array}{l}\text { Random survival forest } \\
\text { algorithm }\end{array}$ & $\begin{array}{l}\text { To develop and validate a } \\
\text { prognostic tool for the } \\
\text { time-to-event risk of major } \\
\text { complications (MCs), } \\
\text { hospital readmission (RA), } \\
\text { and unplanned reoperation } \\
\text { (RO) }\end{array}$ & $\begin{array}{l}\text { Kaplan-Meier estimates showed } \\
\text { that longer duration after operation } \\
\text { frequently accompanied with high risk } \\
\text { of MC }\end{array}$ \\
\hline Yagi (55) & 2018 & $\begin{array}{l}195 \text { surgically } \\
\text { treated ASD } \\
\text { patients }\end{array}$ & $\begin{array}{l}\text { An ensemble of decision } \\
\text { trees utilizing the C5.0 } \\
\text { algorithm with } 5 \text { different } \\
\text { bootstrapped models }\end{array}$ & $\begin{array}{l}\text { To create a predictive model } \\
\text { for complications }\end{array}$ & $\begin{array}{l}92 \% \text { accurate with an AUROC curve } \\
\text { of } 0.963 \text { and } 84 \% \text { accuracy in the } \\
\text { external validation }\end{array}$ \\
\hline
\end{tabular}

Table 1 (continued) 
Table 1 (continued)

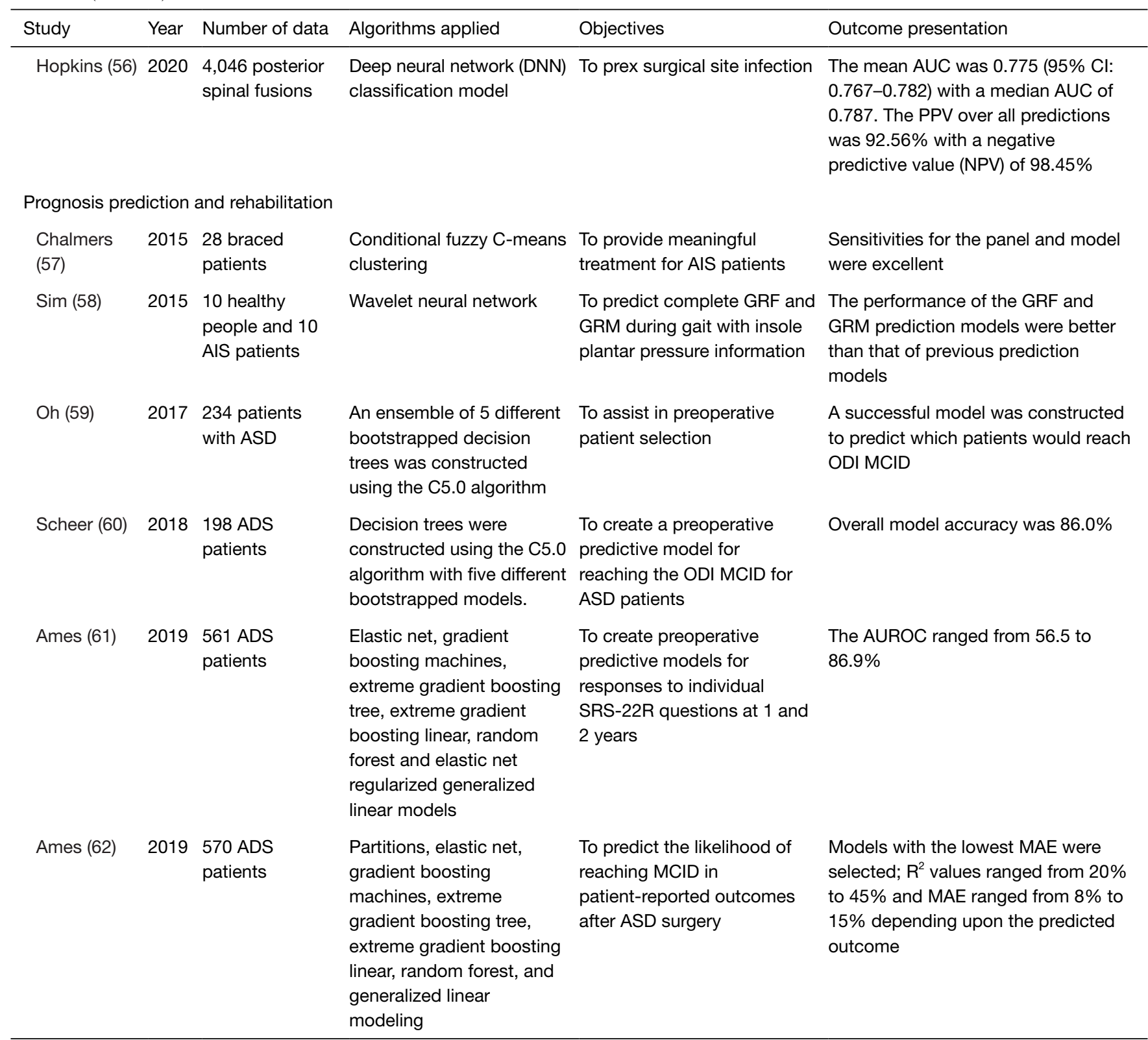

ANN, artificial neural network; AIS, adolescent idiopathic scoliosis; SVM, support vector machine; PPV, positive predictive value; CNN, convolutional neural network; CMCE, circular mean absolute error; ASD, adult spinal deformity; GRF, ground reaction forces; GRM, ground reaction moments; ODI, Oswestry Disability Index; MCID, minimal clinically important difference.

the torso surface. Regarding neuromuscular scoliosis, Bertoncelli et al. (24) applied a predictive model based on a logistic regression (LR) algorithm to predict the probability of scoliosis onset. The predictive accuracy, sensitivity, and specificity of the model were approximately $74 \%$ in full accordance with recent studies applying ML models in clinical fields.
A noninvasive method was gradually developed to assess scoliosis. Jaremko et al. (16) adopted ANNs to quantify torso surface asymmetry, and this method estimated the maximal Cobb angle within $6^{\circ}$ in $63 \%$ of the test data set and was able to distinguish a Cobb angle greater than $30^{\circ}$ with a sensitivity of $100 \%$ and specificity of $75 \%$. However, it is worth mentioning that this research had a 
small data set with only 65 scan-radiograph pairs, which is not very persuasive. An increasing number of harmless screening methods have been developed, and a support vector machine (SVM) classifier has been used to assess the severity of idiopathic scoliosis based on surface topographic images of human backs by Ramirez et al. (17). The results of testing on the dataset showed that the system can achieve $69-85 \%$ accuracy in testing. Yang et al. (30) used unclothed back images with a combination algorithm of Faster-RCNN and Resnet to screen adolescent idiopathic scoliosis (AIS), and this research included images from 3,240 patients. Watanabe et al. (31) created a scoliosis screening system that estimated spinal alignment, the Cobb angle, and vertebral rotation from moiré images based on a convolutional neural network $(\mathrm{CNN})$ to estimate the positions of 12 thoracic and 5 lumbar vertebrae, 17 spinous processes, and the vertebral rotation angle of each vertebra. Using self-contained ultrasound as another detection method for scoliosis, Greer et al. (27) estimated the Cobb angle relative to a vertebrae using a neural network analysis, which was based on a convolutional network and a second fully connected network.

It is important to develop an automatic procedure since measuring the Cobb angle can be time consuming and unreliable. Duong et al. (19) adopted SVM to quantify curve severity with 100 posteroanterior radiographs, and the results were statistically similar $(\mathrm{P}<0.05)$ in $93 \%$ of cases to the manually identified curve. Wu et al. (28) proposed a novel multi-view correlation network (MVCNet) architecture that provided a fully automated end-toend framework for spinal curvature estimation in multiview (both anteroposterior (AP) and lateral (LAT)) X-rays. The results indicated that the MVC-Net's capability of estimating Cobb angles from multi-view $\mathrm{X}$-rays was robust and accurate. Wang et al. (32) also proposed a multi-view extrapolation net (MVE-Net) that provided accurate automated scoliosis estimation from both AP and LAT X-rays. CNN is another suitable ML algorithm for Cobb angle measurement. Galbusera et al. (29) extracted the location of 78 landmarks from three-dimensional reconstructions of 493 spines of patients suffering from various disorders and trained a fully $\mathrm{CNN}$ featuring an additional differentiable spatial to numerical transform (DSNT) layer to predict the location of each landmark. This model automatically determined the shape of the spine in biplanar radiographs and calculated the value of anatomical and posture parameters across a wide range of clinical conditions with robust performance. Horng et al. (33) ever created a CNN system that included three main parts: isolation of the spine, vertebra segmentation, and Cobb angle measurement. Pan et al. (34) proposed two Mask R-CNN models to automatically measure the Cobb angle and diagnose scoliosis on chest $\mathrm{X}$-ray. Jamaludin et al. (35) automated the identification of spinal curvature from total body dual-energy X-ray absorptiometry (DXA) scans using ML techniques, and the final automated model had a sensitivity of $86.5 \%$, specificity of $96.9 \%$, and an area under the curve (AUC) of 0.80 (95\% CI: 0.74-0.87). To dynamically monitor spinal curve progression in AIS, García-Cano et al. (25) proposed a novel approach based on a statistical generative model using random forest regression to predict the shape variation in the spinal curve from the first visit. The estimated shape differed from the real curvature by Cobb angles of $1.83^{\circ}, 5.18^{\circ}$, and $4.79^{\circ}$ in the proximal thoracic, main thoracic, and thoracolumbar/ lumbar sections, respectively.

The primary goal of surgical management of scoliosis is to achieve solid fusion with a well-balanced spine. Insufficient understanding of curve morphology and subsequent improper selection of fusion levels may result in suboptimal outcomes (62). Classification has provided guidance in the treatment of spine deformities. However, though AIS and adult degenerative scoliosis (ADS) are the most common spinal deformities, there can be of great variety in their classification. In this context, identification of AIS classification has been an important topic in the orthopedic community, while ADS classification can be more complex and irregular. Lin et al. (18) implemented a multilayer feed-forward, back-propagation (MLFF/ BP) ANN to test the King classification of scoliosis spinal deformity. The identification rate was $83 \%$ for two hidden layers and $75 \%$ for one hidden layer. Other noninvasive classification methods are also being explored. Adankon et al. (20) divided the 3D image of the surface of the trunk into patches and local geometric descriptors characterizing the back surface of 165 patients with different scoliosis curve types and built a multiclass classifier with leastsquares support vector machines (LS-SVM). The overall accuracy of the system was $95 \%$. For the correct classification rates per class, the results showed $96 \%$, $84 \%$ and $97 \%$ for the thoracic, double major and lumbar/ thoracolumbar curve types, respectively. To solve the problem of low interobserver and intraobserver reliability in AIS classification, García-Cano et al. (26) presented two new techniques to describe the spine, namely, leaveone out and fan leave-one-out for characterizing spine 
curve types, and provided assistance to clinicians in the form of information to classify borderline curvature types. Moreover, a fuzzy logic rule-based classifier was also implemented to classify the spine patterns using the King-Moe classification approach by Birtane et al. (22). As spine deformities are three-dimensional deformities, an increasing number of researchers have paid attention to its 3D classification. Thong et al. (23) adopted an unsupervised clustering method based on stacked autoencoders to simplify the complex nature of $3 \mathrm{D}$ spine models. It is noteworthy that any classification has its advantages and disadvantages, and therefore, it is hard to evaluate which is better. The world is moving into digital archiving, retrieval, and communication of high-resolution images. Menon et al. (21) proposed a newly developed content-based image retrieval (CBIR) software to retrieve images of similar cases of AIS from a database to help plan treatment without adhering to a classification scheme. Although this software did not elaborate a thorough algorithm and the sample size was quite small, this concept is extremely promising for future scoliosis classification.

\section{Surgical decision making}

Classification can truly aid in decision making and instrumentation region selection for surgeons. For example, the Lenke classification (63) provides guidelines for arthrodesis for 6 types and 42 subtypes of AIS patients using expert opinion/consensus and available scientific evidence for clinical decision making; the Scoliosis Research Society (SRS)-Schwab classification (64) and Lenke-Silva classification (65) provide surgical instructions from the perspective of morphology and management for ADS patients. To make this procedure more evidence-based, ML has potential.

Regarding AIS, Mezghani et al. (36) used a database of 1,776 surgically treated AIS cases and investigated a topologically ordered, self-organizing Kohonen network trained using Cobb angle measurements to determine the relationship between the Lenke classification and fusion region selection. The results showed that the recommended fusion region by ML model agreed with the Lenke classification with $88 \%$ overall agreement. Surgery planning could benefit from such map associations by comparing treatment outcomes from similar patients receiving different treatments. Phan et al. (37) used neural networks and Kohonen self-organizing maps (SOM) to classify AIS and conducted a retrospective analysis of AIS curve regions selected for fusion. An AIS SOM with high accuracy was successfully generated. Lenke classification principles were followed in $46 \%$ of the cases but in $82 \%$ of the nodes on the SOM. The SOM highlighted the tendency of surgeons to follow Lenke classification principles for similar curves on the SOM. To identify the range of optimal versus suboptimal rates of spontaneous lumbar Cobb correction (SLCC) and the factors predicting such outcomes in a cohort of Lenke 1 AIS patients, Pasha (40) adopted a decision tree to analyze 71 consecutive AIS patients with a fusion level to L1 and concluded that preoperative lumbar apical vertebrae translation, early postoperative T4-T12 thoracic kyphosis and thoracic apical vertebrae rotation can predict the optimal range of SLCC\%.

As for adult spinal deformities, the principles regarding surgery can be different from AIS and other types of deformities. Health-related quality of life (HRQoL) can be tightly correlated with sagittal alignment. Ames et al. (39) included 570 ADS patients and adopted AI-based hierarchical clustering as a step toward a classification. The results showed that this model identified data patterns that may augment preoperative decision making through the construction of a 2-year risk-benefit grid. Additionally, pattern identification may facilitate treatment optimization by educating surgeons on which treatment patterns yield optimal improvement with the lowest risk. Lafage et al. (38) adopted ML and other types of advanced algorithms to improve surgical outcomes and alignment predictions for surgical planning and prediction of postoperative alignment. These tools, which were able to integrate several parameters and learn from experience, can change the traditional way of selecting treatment and counseling patients. However, the complete algorithm and sample quantity were not mentioned in the article.

\section{Intraoperative manipulation}

To better enhance the surgeon's intraoperative performance, a variety of applications combined with AI were put into routine use. Correct segmentation and vertebral reconstruction are crucial steps in the assessment and management of abnormalities, especially because vertebral rotation is difficult to simulate in spine deformities. Early in 2005, Benameur et al. (41) proposed a hierarchical statistical modeling approach for the unsupervised 3-D biplanar reconstruction of the scoliotic spine. Then, Mirzaalian et al. (42) applied a top-down fully automatic $3 \mathrm{D}$ vertebra segmentation algorithm using global shape- 
related and local appearance-related prior information, in which the latter was handled by a ML-based component. The results indicated a symmetric point-to-mesh surface error of $1.37 \pm 0.37 \mathrm{~mm}$, which matched the current stateof-the-art methods. In 2018, Zareie et al. (66) compared a multilayer perceptron neural network (MLPNN) and a newly developed adaptive pulse coupled neural network (APCNN) in the automatic segmentation of vertebrae in 3D CT images and concluded that the performance of the presented APCNN-based algorithm was dominant.

Pedicle location and recognition play important roles in screw implantation for spine deformities. Huo et al. (48) proposed a method based on 400 individual vertebral models to automatically recognize the vertebral pedicle in individual vertebral models and draw pedicle contours. The procedure included three steps: first, the individual vertebral models were preprocessed to obtain their point clouds; then, a modified Point-Net model was used to segment the pedicle areas from the individual vertebral point clouds; afterwards, the segmentation results were used to automatically fit the cross-sections of pedicles and finally generate the pedicle contours as surgical references. For most scoliosis cases, vertebrae often have axial rotation, and this point should be taken into great consideration when inserting pedicle screws. Ebrahimi et al. (47) included a total of 149 healthy and AIS subjects and developed an automated pedicle detector based on image analysis, ML and fast manual identification of a few landmarks to calculate vertebrae axial rotation values in frontal radiographs with minimal user intervention and robust quasi-automated pedicle localization. Minimally invasive surgery is another promising strategy for spine deformities; therefore, accurate percutaneous spinal needle insertion procedures are necessary. Hetherington et al. (45) developed a real-time system based on deep CNN to classify transverse images of the lower spine, and this method might contribute to the development of a minimally invasive treatment for ADS. To satisfy the goal of verification, screw insertion was performed intraoperatively. Esfandiari et al. (46) found that the CNN framework was capable of segmenting screw shafts with $93 \%$ and $83 \%$ accuracy when tested on synthetic X-rays and on clinically realistic $\mathrm{X}$-rays, respectively. To achieve the goal of multiobjective optimization design of spinal pedicle screws in the treatment of deformity corrections, Amaritsakul et al. (43) found that the hybrid of ANN and genetic algorithm (GA) was ideal with simultaneous high bending and pullout performances. Moreover, automatic matching of surgeries to predict the surgeons' next actions can be helpful for spine deformity surgeons. For this purpose, Forestier et al. (44) proposed an efficient algorithm to find the optimal partial alignment and a prediction system using maximum a posteriori probability estimation and filtering in lumbar disc herniation removal and anterior cervical discectomy. The results showed that this method outperformed the state-ofthe-art methods by predicting the next task that the surgeon will perform with $95 \%$ accuracy. We believe this kind of mode can be extended to orthopedic surgeries for spine deformities in the near future.

\section{Complication predictions}

The definition of complication in this review was defined as adverse events that can significantly affect the patients' quality of life and frequently required clinical intervention during the perioperative period. The goal of surgical treatment for patients with scoliosis is undoubtedly to improve their HRQoL, and thus related complications should be carefully taken into consideration, especially in adult spinal deformity (ASD) patients. Any unexpected complication may cause miserable results, and it is of great importance to predict these problems before performing surgery. Normally, few complications are found in the AIS population, and nearly all the references regarding scoliosis and ML are about ADS. Pellisé et al. (53) aimed to assess the incidence of adverse events after ASD surgery and to develop and validate a prognostic tool for the time-to-event risk of major complications (MCs), hospital readmission (RA), and unplanned reoperation (RO). They created a random survival forest algorithm, and the results showed that surgical invasiveness, age, magnitude of deformity, and frailty were the strongest predictors of MCs. Individual cumulative risk estimates at 2 years ranged from $3.9 \%$ to $74.1 \%$ for MCs, from $3.17 \%$ to $44.2 \%$ for RAs, and from $2.67 \%$ to $51.9 \%$ for ROs. Scheer et al. (50) performed similar studies with ASD patients, and they constructed an ensemble of decision trees utilizing the C5.0 algorithm with 5 different bootstrapped models. With a sample of 557 ADS patients, the overall model accuracy was 87.6\% correct, with an AUC of the receiver operating characteristic (AUROC) curve of 0.89 indicating a very good model fit. They reported that twenty variables were determined to be the top predictors. Kim et al. (51) created models using the American Society of Anesthesiologists classification (ASA class) as a benchmark for prediction of cardiac complications, wound complications, venous 
thromboembolism (VTE), and mortality in ASD patients. The results showed that the ANN outperformed LR in predicting cardiac complications, wound complications, and mortality $(\mathrm{P}<0.05)$. To analyze MC 2 years after corrective spine surgery for ASD, Yagi et al. (52) constructed decisionmaking tree models using spinal alignment, demographic data, and surgical invasiveness. The test samples showed that their predictive model was $92 \%$ accurate with an AUROC curve of 0.963 and $84 \%$ accuracy in the external validation. As patients with spine deformities often experience long and deep wounds, postoperative surgical site infection is a common complication. Hopkins et al. (55) trained a deep neural network classification model using 35 unique input variables in a retrospective cohort of 4,046 posterior spinal fusions. The overall rate of infection was $1.5 \%$. The mean AUC, representing the accuracy of the model, across all 300 iterations was 0.775 (95\% CI: $0.767-$ 0.782) with a median AUC of 0.787 . Although this research did not focus on scoliosis, the application of ML can be examined in future studies.

Regarding radiographic complications, especially proximal junctional kyphosis (PJK) and proximal junctional failure (PJF) in ASD patients, there were only two studies reported to date. Scheer et al. (49) constructed an ensemble of decision trees using the C5.0 algorithm with 5 different bootstrapped models in a cohort of 510 ASD patients, and the overall model accuracy was $86.3 \%$, with an AUC of 0.89 indicating a good model fit. Their study listed the 7 strongest (importance $\geq 0.95$ ) predictors: age, lowermost instrumented vertebra (LIV), preoperative sagittal vertical axis (SVA), uppermost instrumented vertebra (UIV) implant type, UIV, preoperative pelvic tilt (PT), and preoperative pelvic incidence and lumbar lordosis (PI-LL). In 2018, Yagi et al. (54) fine-tuned the predictive model for PJF with the construction of decision-making trees using the C5.0 algorithm with 10 different bootstrapped models and assessed performance with 145 surgically treated ASD patients. Their predictive model was $100 \%$ accurate in the testing samples with an AUC of 1.0, indicating excellent fit. The best predictors were (strongest to weakest): PT, bone mineral density (BMD), LIV level (pelvis), UIV level (lower thoracic), pedicle subtraction osteotomy (PSO), global alignment, body mass index (BMI), PI-LL, and age.

\section{Prognosis prediction and rehabilitation}

All surgeons crave for a better clinical efficacy for their patients; however, the prognosis across differing circumstances can be quite different. Therefore, prognosis prediction is necessary, and currently, different ML approaches are being explored according to their specific characteristics. Regarding functional indices, Scheer et al. (59) constructed decision trees using the C5.0 algorithm with five different bootstrapped models according to baseline demographic, radiographic, HRQoL, and surgical factors to predict patients meeting the Oswestry Disability Index (ODI) minimal clinically important difference (MCID) at the two-year postoperative followup. The overall model accuracy was $86.0 \%$, with an AUC of 0.94 , and the top 11 predictors for achieving the MCID were listed. Ames et al. (60) compared eight predictive algorithms using 75 variables of demographics, baseline patient-reported outcomes (PROs), and modifiable surgical parameters at four time horizons: preoperative or postoperative baseline to 1 year and preoperative or postoperative baseline to 2 years. They concluded that patients with worse preoperative baseline PROs tended to achieve clinically relevant improvements. This team also developed six different prediction algorithms for all the individual questions on the SRS-22R after ASD surgery directed toward individualized medicine, and the AUROC ranged from $56.5 \%$ to $86.9 \%$, reflecting successful fits for most questions (61). Oh et al. (58) aimed to create a validated MCID model that had the potential to assist in patient selection, thereby improving outcomes. An ensemble of 5 different bootstrapped decision trees was constructed using the C5.0 algorithm with $85.5 \%$ accuracy and 0.96 AUC.

Regarding rehabilitation, Chalmers et al. (56) compared human experts' and a fuzzy model's predictions of outcomes of scoliosis bracing treatment. The model was constructed using conditional fuzzy C-means clustering to discover patterns in retrospective patient data and was capable of providing meaningful brace treatment recommendations. A wavelet neural network was adopted to predict complete ground reaction forces and moments during gait with insole plantar pressure information by $\operatorname{Sim}$ et al. (57), and the results might help improve the gait of AIS patients. In addition, a robotic spine exoskeleton (67) capable of controlling the position/orientation of specific cross-sections of the human torso while simultaneously measuring the forces/moments exerted on the body opened the possibility for the design of spine braces incorporating patient-specific torso stiffness characteristics and the potential for new interventions using the dynamic modulation of 3-D forces for scoliosis treatment. Additionally, robotic rehabilitation 
of the upper extremity after neurological injury (68) and trunk robot rehabilitation training with active stepping after trunk motor cortex injury (69) might be promising assistant rehabilitation methods in the near future.

\section{Current limitations and future directions}

The implementation of ML technologies for scoliosis, especially regarding tools with a direct clinical impact, is undoubtedly contributing to a paradigm shift. However, with the advent of new techniques, the current limitations and ethical problems should be reasonably considered. A systematic review in neurosurgical literature concluded that in spite of great potential of ML models in augmenting the decision-making capacity of clinicians in neurosurgical applications, significant hurdles remain associated with creating, validating, and deploying ML models in the clinical setting (1). Perhaps the most barrier regarding $\mathrm{ML}$ adoption is the lack of robust frameworks used to assess the performance and development of the related algorithms. Furthermore, the mechanisms driving the algorithms is sometimes complicated and even unpractical. In fact, there is an absence of clear gold criteria for ML models in addressing clinical problems. Regarding aspects of disease screening, diagnosis and classification, ML still shows the phenomena of misdiagnosis and missed diagnosis, and its accuracy is not $100 \%$ effective. Although there are many types and theories as support, many details remain to be optimized for more thorough and further simulation and planning. In addition, although ML is much more improved than simple mathematical statistics, the basic principle appears as a black box to an external user, and its predictions largely appear to be determined by an obscure logic that cannot be understood or interpreted by a human observer $(70,71)$. Secondly, it is relatively difficult to collect high-volume patients' date for a single-center institution due to privacy considerations or across institutions. The deployment of such powerful technologies in the area of scoliosis is still in its infancy, and scoliosis is a relatively narrow branch of the spine field; the available number of samples is not as sufficient as other subjects. Thirdly, another important preconception regarding the role of ML models in clinical spine realm is that the status of clinicians could be shaken, a so-called term 'human-vsmachine' (72). In clinical practice, although ML could give high analysis accuracy in clinical guidance, the clinicians still must pay attention to the implications of this analysis (73). Researchers apply a variety of algorithms to develop applications, and the annotation and collection of the original data should be rigorous. Before extension to other surgeons, the application should require more comprehensive testing and verification with respect to other technologies. When applied in the real world, the surgeons' role in the final decision should still be emphasized (74). Last but not least, ethical problems should never be neglected with ML in the scoliosis field. Data privacy and security remain a problem due to the massive amount of clinical and imaging data required, thus issues about data collection, transmission and storage, as well as informed consent are involved (2). Data anonymization is commonly advocated; nevertheless, patients retain rights to their anonymized data, which are subjected to strict regulations about storage, transmission and use, especially when data are used in a for-profit environment (75).

Consequently, conducting a high-quality study may be restricted. Also, the ability to evaluate the study design such as power analysis can be limited. Therefore, it is imperative to reach a consensus regarding the optimal method standardization of ML in clinical practice, and a high-quality study taking human-and -machine approach may reveal how the clinicians can benefit from ML models. The following suggestion recommendations should be considered by clinicians when ML is applied in scoliosis. Firstly, a multidisciplinary team containing spine clinicians, engineers, statistical experts, and data scientists should be created to evaluate the ML tools, which could compensate to the knowledge limitations for any single team. Secondly, there was considerable heterogeneity in the modeling methods used, including the inclusion criteria, input and output variables used, and so on. The comparison of different neural network algorithms could assist in selecting the best model with the best performance. Additionally, the advantages and disadvantages of different algorithms for each spinal disorder should also be considered (76). Therefore, future study should focus on the validation of ML models on heterogeneous test sets prior to deployment and the regulation of ML performance after deployment in clinical practice. Thirdly, concept shifting from human $v$ s. machine to human-and-machine may be essential to over these barriers. Take an example, there were four studies concluded that ML combining with clinical decision making is superior to ML models or clinical decision making alone (72,76-78). Using the experience of clinicians as a prerequirement, the application of ML in spine deformity, such as scoliosis, will be more promising in increasing the accessibility of clinical data. 


\section{Conclusions}

Currently, ML in practice is still an art of weak AI. Although we presented its application in different clinical practice stages, there is still a lack of a complete cycle of the clinical procedure that can help surgeons make decisions from diagnosis to prognosis. The related applications of ML on etiology (79), gait analysis (57) and electronic medical records analysis (80) should also be continued. We believe that the implementation of sophisticated ML for scoliosis promises a revolution in how surgeons perform throughout all aspects of the clinical practice related to scoliosis (14). More effective and reliable predictive models for new ways of collecting, accessing, sharing, storing, analyzing and presenting the data need further exploration.

\section{Acknowledgments}

Funding: This study was supported by Chinese National Natural Science Fund (31870985).

\section{Footnote}

Reporting Checklist: The authors have completed the Narrative Review reporting checklist. Available at http:// dx.doi.org/10.21037/atm-20-5495

Conflicts of Interest: All authors have completed the ICMJE uniform disclosure form (available at http://dx.doi. org/10.21037/atm-20-5495). The authors have no conflicts of interest to declare.

Ethical Statement: The authors are accountable for all aspects of the work in ensuring that questions related to the accuracy or integrity of any part of the work are appropriately investigated and resolved.

Open Access Statement: This is an Open Access article distributed in accordance with the Creative Commons Attribution-NonCommercial-NoDerivs 4.0 International License (CC BY-NC-ND 4.0), which permits the noncommercial replication and distribution of the article with the strict proviso that no changes or edits are made and the original work is properly cited (including links to both the formal publication through the relevant DOI and the license). See: https://creativecommons.org/licenses/by-nc-nd/4.0/.

\section{References}

1. Machine learning and artificial intelligence research for patient benefit: 20 critical questions on transparency, replicability, ethics, and effectiveness. BMJ 2020;369:m1312.

2. Galbusera F, Casaroli G, Bassani T. Artificial intelligence and machine learning in spine re-search. JOR Spine 2019;2:e1044.

3. Walsh SLF, Humphries SM, Wells AU, et al. Imaging research in fibrotic lung disease; ap-plying deep learning to unsolved problems. Lancet Respir Med 2020;8:1144-53.

4. Morley J, Floridi L. An ethically mindful approach to AI for health care. Lancet 2020;395:254-5.

5. Poduval M, Ghose A, Manchanda S, et al. Artificial Intelligence and Machine Learning: A New Disruptive Force in Orthopaedics. Indian J Orthop 2020;54:109-22.

6. Nagendran M, Chen Y, Lovejoy CA, et al. Artificial intelligence versus clinicians: systematic review of design, reporting standards, and claims of deep learning studies. BMJ 2020;368:m689.

7. Jamaludin A, Kadir T, Zisserman A. SpineNet: Automated classification and evidence visualization in spinal MRIs. Medical Image Analysis 2017;41:63-73.

8. Jamaludin A, Lootus M, Kadir T, et al. ISSLS PRIZE IN BIOENGINEERING SCIENCE 2017: Automation of reading of radiological features from magnetic resonance images (MRIs) of the lumbar spine without human intervention is comparable with an expert radiologist. Eur Spine J 2017;26:1374-83.

9. Goyal A, Ngufor C, Kerezoudis P, et al. Can machine learning algorithms accurately predict discharge to nonhome facility and early unplanned readmissions following spinal fusion? Analysis of a national surgical registry. J Neurosurg Spine 2019:1-11.

10. Arvind V, Kim JS, Oermann EK, et al. Predicting Surgical Complications in Adult Patients Undergoing Anterior Cervical Discectomy and Fusion Using Machine Learning. Neurospine 2018;15:329-37.

11. Kim JS, Merrill RK, Arvind V, et al. Examining the Ability of Artificial Neural Networks Machine Learning Models to Accurately Predict Complications Following Posterior Lumbar Spine Fusion. Spine 2018;43:853-60.

12. Wirries A, Geiger F, Hammad A, et al. Artificial intelligence facilitates decision-making in the treatment of lumbar disc herniations. Eur Spine J 2020. [Epub ahead of print]. doi: 10.1007/s00586-020-06613-2. 
13. Joshi RS, Haddad AF, Lau D, et al. Artificial Intelligence for Adult Spinal Deformity. Neurospine 2019;16:686-94.

14. Joshi RS, Lau D, Ames CP. Artificial Intelligence and the Future of Spine Surgery. Neurospine 2019;16:637-9.

15. Jaremko J, Delorme S, Dansereau J, et al. Use of Neural Networks to Correlate Spine and Rib Deformity in Scoliosis. Comput Methods Biomech Biomed Engin 2000,3:203-13.

16. Jaremko JL, Poncet P, Ronsky J, et al. Estimation of spinal deformity in scoliosis from torso surface cross sections. Spine (Phila Pa 1976) 2001;26:1583-91.

17. Ramirez L, Durdle NG, Raso VJ, et al. A support vector machines classifier to assess the severity of idiopathic scoliosis from surface topography. IEEE Trans Inf Technol Biomed 2006;10:84-91.

18. Lin H. Identification of spinal deformity classification with total curvature analysis and artificial neural network. IEEE Transactions on Bio-Medical Engineering 2008,55:376-82.

19. Duong L, Cheriet F, Labelle H. Automatic detection of scoliotic curves in posteroanterior radiographs. IEEE Trans Biomed Eng 2010;57:1143-51.

20. Adankon MM, Dansereau J, Labelle H, et al. Non invasive classification system of scoliosis curve types using least-squares support vector machines. Artif Intell Med 2012;56:99-107.

21. Menon KV, Kumar D, Thomas T. Experiments with a novel content-based image retrieval software: can we eliminate classification systems in adolescent idiopathic scoliosis? Global Spine J 2014;4:13-20.

22. Birtane S, Korkmaz H. Rule-based fuzzy classifier for spinal deformities. Biomed Mater Eng 2014;24:3311-9.

23. Thong $\mathrm{W}$, Parent S, Wu J, et al. Three-dimensional morphology study of surgical adolescent idiopathic scoliosis patient from encoded geometric models. Eur Spine J 2016;25:3104-13.

24. Bertoncelli CM, Bertoncelli D, Elbaum L, et al. Validation of a Clinical Prediction Model for the Development of Neuromuscular Scoliosis: A Multinational Study. Pediatr Neurol 2018;79:14-20.

25. García-Cano E, Arámbula Cosío F, Duong L, et al. Prediction of spinal curve progression in Adolescent Idiopathic Scoliosis using Random Forest regression. Comput Biol Med 2018;103:34-43.

26. García-Cano E, Arámbula Cosío F, Duong L, et al. Dynamic ensemble selection of learn-er-descriptor classifiers to assess curve types in adolescent idiopathic scoliosis. Med Biol Eng Comput 2018;56:2221-31.

27. Greer H, Gerber S, Niethammer M, et al. Scoliosis screening and monitoring using self contained ultrasound and neural networks. Proc IEEE Int Symp Biomed Imaging 2018;2018:1500-3.

28. Wu H, Bailey C, Rasoulinejad P, et al. Automated comprehensive Adolescent Idiopathic Scoliosis assessment using MVC-Net. Med Image Anal 2018;48:1-11.

29. Galbusera F, Niemeyer F, Wilke HJ, et al. Fully automated radiological analysis of spinal disorders and deformities: a deep learning approach. Eur Spine J 2019;28:951-60.

30. Yang J, Zhang K, Fan H, et al. Development and validation of deep learning algorithms for scoliosis screening using back images. Commun Biol 2019;2:390.

31. Watanabe K, Aoki Y, Matsumoto M. An Application of Artificial Intelligence to Diagnostic Imaging of Spine Disease: Estimating Spinal Alignment From Moiré Images. Neurospine 2019;16:697-702.

32. Wang L, Xu Q, Leung S, et al. Accurate automated Cobb angles estimation using multi-view extrapolation net. Med Image Anal 2019;58:101542.

33. Horng MH, Kuok CP, Fu MJ, et al. Cobb Angle Measurement of Spine from X-Ray Images Using Convolutional Neural Network. Comput Math Methods Med 2019;2019:6357171.

34. Pan Y, Chen Q, Chen T, et al. Evaluation of a computeraided method for measuring the Cobb angle on chest X-rays. Eur Spine J 2019;28:3035-43.

35. Jamaludin A, Fairbank J, Harding I, et al. Identifying Scoliosis in Population-Based Cohorts: Automation of a Validated Method Based on Total Body Dual Energy X-ray Absorp-tiometry Scans. Calcif Tissue Int 2020;106:378-85.

36. Mezghani N, Phan P, Mitiche A, et al. A Kohonen neural network description of scoliosis fused regions and their corresponding Lenke classification. Int J Comput Assist Radiol Surg 2012;7:257-64.

37. Phan P, Mezghani N, Wai EK, et al. Artificial neural networks assessing adolescent idiopathic scoliosis: comparison with Lenke classification. Spine J 2013;13:1527-33.

38. Lafage R, Pesenti S, Lafage V, et al. Self-learning computers for surgical planning and prediction of postoperative alignment. Eur Spine J 2018;27:123-8.

39. Ames CP, Smith JS, Pellisé F, et al. Artificial Intelligence Based Hierarchical Clustering of Patient Types and Intervention Categories in Adult Spinal Deformity Surgery: Towards a New Classification Scheme that Predicts Quality and Value. Spine (Phila Pa 1976) 2019;44:915-26.

40. Pasha S, Mac-Thiong JM. Defining criteria for optimal 
lumbar curve correction following the selective thoracic fusion surgery in Lenke 1 adolescent idiopathic scoliosis: developing a decision tree. Eur J Orthop Surg Traumatol 2020;30:513-22.

41. Benameur S, Mignotte M, Labelle H, et al. A hierarchical statistical modeling approach for the unsupervised 3-D biplanar reconstruction of the scoliotic spine. IEEE Trans Biomed Eng 2005;52:2041-57.

42. Mirzaalian H, Wels M, Heimann T, et al. Fast and robust $3 \mathrm{D}$ vertebra segmentation using statistical shape models. Annu Int Conf IEEE Eng Med Biol Soc 2013;2013:3379-82.

43. Amaritsakul Y, Chao CK, Lin J. Multiobjective optimization design of spinal pedicle screws using neural networks and genetic algorithm: mathematical models and mechanical validation. Comput Math Methods Med 2013;2013:462875.

44. Forestier G, Petitjean F, Riffaud L, et al. Automatic matching of surgeries to predict surgeons' next actions. Artif Intell Med 2017;81:3-11.

45. Hetherington J, Lessoway V, Gunka V, et al. SLIDE: automatic spine level identification system using a deep convolutional neural network. Int J Comput Assist Radiol Surg 2017;12:1189-98.

46. Esfandiari H, Newell R, Anglin C, et al. A deep learning framework for segmentation and pose estimation of pedicle screw implants based on C-arm fluoroscopy. Int J Comput Assist Radiol Surg 2018;13:1269-82.

47. Zareie M, Parsaei H, Amiri S, et al. Automatic segmentation of vertebrae in 3D CT images using adaptive fast 3D pulse coupled neural networks. Australas Phys Eng Sci Med 2018;41:1009-20.

48. Ebrahimi S, Gajny L, Vergari C, et al. Vertebral rotation estimation from frontal $\mathrm{X}$-rays using a quasi-automated pedicle detection method. Eur Spine J 2019;28:3026-34.

49. Huo X, Wang H, Shao K, et al. Automatic generation of pedicle contours in $3 \mathrm{D}$ vertebral models. Comput Biol Med 2020;116:103565.

50. Scheer JK, Osorio JA, Smith JS, et al. Development of Validated Computer-based Pre-operative Predictive Model for Proximal Junction Failure (PJF) or Clinically Significant PJK With 86\% Accuracy Based on 510 ASD Patients With 2-year Follow-up. Spine (Phila Pa 1976) 2016;41:E1328-35.

51. Scheer JK, Smith JS, Schwab F, et al. Development of a preoperative predictive model for major complications following adult spinal deformity surgery. J Neurosurg Spine 2017;26:736-43.
52. Kim JS, Arvind V, Oermann EK, et al. Predicting Surgical Complications in Patients Undergoing Elective Adult Spinal Deformity Procedures Using Machine Learning. Spine De-form 2018;6:762-70.

53. Yagi M, Hosogane N, Fujita N, et al. Predictive model for major complications 2 years after corrective spine surgery for adult spinal deformity. Eur Spine J 2019;28:180-7.

54. Pellisé F, Serra-Burriel M, Smith JS, et al. Development and validation of risk stratification models for adult spinal deformity surgery. J Neurosurg Spine 2019:1-13.

55. Yagi M, Fujita N, Okada E, et al. Fine-tuning the Predictive Model for Proximal Junctional Failure in Surgically Treated Patients With Adult Spinal Deformity. Spine (Phila Pa 1976) 2018;43:767-73.

56. Hopkins BS, Mazmudar A, Driscoll C, et al. Using artificial intelligence (AI) to predict postoperative surgical site infection: A retrospective cohort of 4046 posterior spinal fusions. Clin Neurol Neurosurg 2020;192:105718.

57. Chalmers E, Pedrycz W, Lou E. Human experts' and a fuzzy model's predictions of out-comes of scoliosis treatment: a comparative analysis. IEEE Trans Biomed Eng 2015;62:1001-7.

58. Sim T, Kwon H, Oh SE, et al. Predicting Complete Ground Reaction Forces and Moments During Gait With Insole Plantar Pressure Information Using a Wavelet Neural Network. J Biomech Eng 2015;137.

59. Oh T, Scheer JK, Smith JS, et al. Potential of predictive computer models for preoperative patient selection to enhance overall quality-adjusted life years gained at 2-year follow-up: a simulation in 234 patients with adult spinal deformity. Neurosurg Focus 2017;43:E2.

60. Scheer JK, Osorio JA, Smith JS, et al. Development of a Preoperative Predictive Model for Reaching the Oswestry Disability Index Minimal Clinically Important Difference for Adult Spinal Deformity Patients. Spine Deform 2018;6:593-9.

61. Ames CP, Smith JS, Pellisé F, et al. Development of Deployable Predictive Models for Minimal Clinically Important Difference Achievement Across the Commonly Used Health-related Quality of Life Instruments in Adult Spinal Deformity Surgery. Spine (Phila Pa 1976) 2019;44:1144-53.

62. Ames CP, Smith JS, Pellisé F, et al. Development of predictive models for all individual questions of SRS22R after adult spinal deformity surgery: a step toward individualized medicine. Eur Spine J 2019;28:1998-2011.

63. Kang DG, Lehman RA, Lenke LG. Challenges in the classification of adolescent idiopathic scoliosis and the utility 
of artificial neural networks. Spine J 2013;13:1534-7.

64. Lenke LG, Betz RR, Harms J, et al. Adolescent idiopathic scoliosis: a new classification to determine extent of spinal arthrodesis. J Bone Joint Surg Am 2001;83:1169-81.

65. Schwab F, Ungar B, Blondel B, et al. Scoliosis Research Society-Schwab adult spinal deformity classification: a validation study. Spine (Phila Pa 1976) 2012;37:1077-82.

66. Silva FE, Lenke LG. Adult degenerative scoliosis: evaluation and management. Neuro-surgical Focus 2010,28:E1.

67. Park JH, Stegall PR, Roye DP, et al. Robotic Spine Exoskeleton (RoSE): Characterizing the 3-D Stiffness of the Human Torso in the Treatment of Spine Deformity. IEEE Trans Neural Syst Rehabil Eng 2018;26:1026-35.

68. Sharp KG, Duarte JE, Gebrekristos B, et al. Robotic Rehabilitator of the Rodent Upper Extremity: A System and Method for Assessing and Training Forelimb Force Production after Neurological Injury. J Neurotrauma 2016;33:460-7.

69. Oza CS, Giszter SF. Trunk robot rehabilitation training with active stepping reorganizes and enriches trunk motor cortex representations in spinal transected rats. J Neurosci 2015;35:7174-89.

70. Pesapane F, Volonté C, Codari M, et al. Artificial intelligence as a medical device in radiology: ethical and regulatory issues in Europe and the United States. Insights Imaging 2018;9:745-53.

71. Char DS, Shah NH, Magnus D. Implementing Machine Learning in Health Care - Ad-dressing Ethical Challenges. N Engl J Med 2018;378:981-3.

Cite this article as: Chen $\mathrm{K}$, Zhai X, Sun K, Wang H, Yang C, Li M. A narrative review of machine learning as promising revolution in clinical practice of scoliosis. Ann Transl Med 2021;9(1):67. doi: 10.21037/atm-20-5495
72. Senders JT, Arnaout O, Karhade AV, et al. Natural and Artificial Intelligence in Neuro-surgery: A Systematic Review. Neurosurgery 2018;83:181-92.

73. Kedra J, Gossec L. Big Data and artificial intelligence: Will they change our practice? Joint Bone Spine 2020;87:107-9.

74. Mazzanti M, Shirka E, Gjergo H, et al. Imaging, Health Record, and Artificial Intelligence: Hype or Hope? Curr Cardiol Rep 2018;20:48.

75. Winfield AFT, Mathematical Jirotka M. Ethical governance is essential to building trust in robotics and artificial intelligence systems. Philos Trans A Math Phys Eng Sci 2018;376.

76. Azimi P, Yazdanian T, Benzel EC, et al. A Review on the Use of Artificial Intelligence in Spinal Diseases. Asian Spine J 2020;14:543-71.

77. Kerr WT, Nguyen ST, Cho AY, et al. Computer-Aided Diagnosis and Localization of Lateralized Temporal Lobe Epilepsy Using Interictal FDG-PET. Front Neurol 2013;4:31.

78. Abdolmaleki P, Mihara F, Masuda K, et al. Neural networks analysis of astrocytic gliomas from MRI appearances. Cancer Lett 1997;118:69-78.

79. Waller T, Nowak R, Tkacz M, et al. Familial or Sporadic Idiopathic Scoliosis - classification based on artificial neural network and GAPDH and ACTB transcription profile. Biomed Eng Online 2013;12:1.

80. Schwartz JT, Gao M, Geng EA, et al. Applications of Machine Learning Using Electronic Medical Records in Spine Surgery. Neurospine 2019;16:643-53. 